ORIGINAL ARTICLE

\title{
Effect of anticipation during unknown or unexpected exercise duration on rating of perceived exertion, affect, and physiological function
}

\author{
D A Baden, T L McLean, R Tucker, T D Noakes, A St Clair Gibson
}

Br J Sports Med 2005;39:742-746. doi: 10.1136/bjsm.2004.016980

See end of article for authors' affiliations

Correspondence to:

Professor St Clair Gibson University of Cape Town,

Research Unit for Exercise Science and Sports Medicine, Newlands 7725 , South Africa: agibson@sports.uct.ac.za

Accepted 21 March 2005
Objectives: To determine the effect of unknown exercise duration and an unexpected increase in exercise duration on rating of perceived exertion (RPE), affect, and running economy during treadmill running. Methods: Sixteen well trained male and female runners completed three bouts of treadmill running at $75 \%$ of their peak treadmill running speed. In the first trial, they were told to run for 20 minutes and were stopped at 20 minutes (20 MIN). In another trial, they were told to run for 10 minutes, but at 10 minutes were told to run for a further 10 minutes $(10 \mathrm{MIN})$. In the final trial, they were not told for how long they would be running but were stopped after 20 minutes (unknown, UN). During each of the running bouts, $\mathrm{RPE}$, oxygen consumption $(\mathrm{ml} / \mathrm{kg} / \mathrm{min})$, heart rate (beats $/ \mathrm{min})$, stride frequency $\left(\mathrm{min}^{-1}\right)$, affect scores (arbitrary units), and attentional focus (percentage associative thought scores) were recorded.

Results: RPE increased significantly between 10 and 11 minutes in the $10 \mathrm{MIN}$ compared with the $20 \mathrm{MIN}$ and UN trials $(p<0.05)$. The affect score decreased significantly between 10 and 11 minutes in the $10 \mathrm{MIN}$ compared with the $20 \mathrm{MIN}$ trial $(p<0.05)$. Running economy, as measured by oxygen consumption, was significantly lower in the UN compared with the $20 \mathrm{MIN}$ trial from 10 to 19 minutes $(p<0.05)$.

Conclusions: The change in RPE between 10 and 11 minutes in the $10 \mathrm{MIN}$ trial suggests that RPE is not purely a measure of physical exertion, as treadmill speed was maintained at a constant pace both before and after the unexpected increase in exercise duration. The associated changes in affect score at similar times in the 10 MIN trial supports the hypothesis that RPE has an affective component.
$\mathrm{T}$ he rating of perceived exertion (RPE) during exercise, measured using the Borg 15 point scale ${ }^{12}$ or category ratio scale, ${ }^{2}$ has been causally linked to physiological variables such as muscle force output, ${ }^{3}$ heart rate, ventilation, respiratory rate, oxygen uptake, and blood lactate concentrations. ${ }^{4}$

However, psychological factors also appear to contribute to RPE. ${ }^{5}{ }^{6}$ Rejeski and Ribisl ${ }^{5}$ found that when subjects were instructed to run for 30 minutes, their RPE was lower throughout the run than when they were instructed to run for 20 minutes, despite running at the same intensity in both conditions.

Affect may be one psychological factor contributing to RPE. Affect refers to a short duration, high intensity emotion or feeling related to an idea or object. ${ }^{7}$ Hardy and Rejeski ${ }^{8}$ measured RPE and affect using an affect (feeling) scale ${ }^{9}$ during exercise at different intensities. A significant negative correlation between RPE and affect score was found at various exercise intensities, with stronger correlations at higher intensities.

Knowledge of exercise duration has also been suggested to play an important role in the control of physiological and psychological resources during exercise. ${ }^{10-13}$ Studies have shown that expectation of task duration and anticipation of an end point influence subjective ratings of fatigue, ${ }^{6} \mathrm{RPE}^{5}$ associative thought processes, ${ }^{11}$ and neuromuscular efficiency. ${ }^{14}$

Therefore, although studies have suggested that perceived exertion may be influenced by both affect and knowledge of exercise duration, the relation between perceived exertion, affect, and knowledge of exercise duration has not been well described. The aim of this study was therefore to investigate how the anticipation of exercise duration influenced RPE, affect, and running economy, as measured by changes in oxygen consumption $\left(\mathrm{VO}_{2}\right)$ during the exercise bout, and, in particular, how these variables were affected when the actual exercise duration was different from that anticipated before the onset of the exercise bout.

\section{METHODS \\ Subjects}

Sixteen subjects (eight male, eight female) were recruited from local running clubs. All subjects were healthy, moderately trained, and fully informed of the risks associated with the study. The research and ethics committee of the Faculty of Health Sciences, University of Cape Town approved the study.

\section{Familiarisation session and determination of peak} treadmill running speed

Age, height, mass, body fat percentage, ${ }^{15}$ and training history were recorded for each subject at their first visit to the laboratory. During this session, subjects were informed that they would perform three trials during which running on the treadmill would last a maximum of 30 minutes. They then performed a peak treadmill running speed (PTRS) test using a protocol described previously, ${ }^{16}$ which was used to calculate maximal aerobic capacity $\left(\mathrm{VO}_{2}\right.$ peak $)$ and the treadmill speed to be used for subsequent experimental trials (described below). This session was also an opportunity for the subjects to become familiar with running on the treadmill, as not all of them ran regularly on a treadmill as part of their training. Finally, the subjects were also familiarised with the three scales to be used in the subsequent experimental trials (described below).

Abbreviations: PTRS, peak treadmill running speed; RPE, rating of perceived exertion; $\mathrm{VO}_{2}$, oxygen consumption 


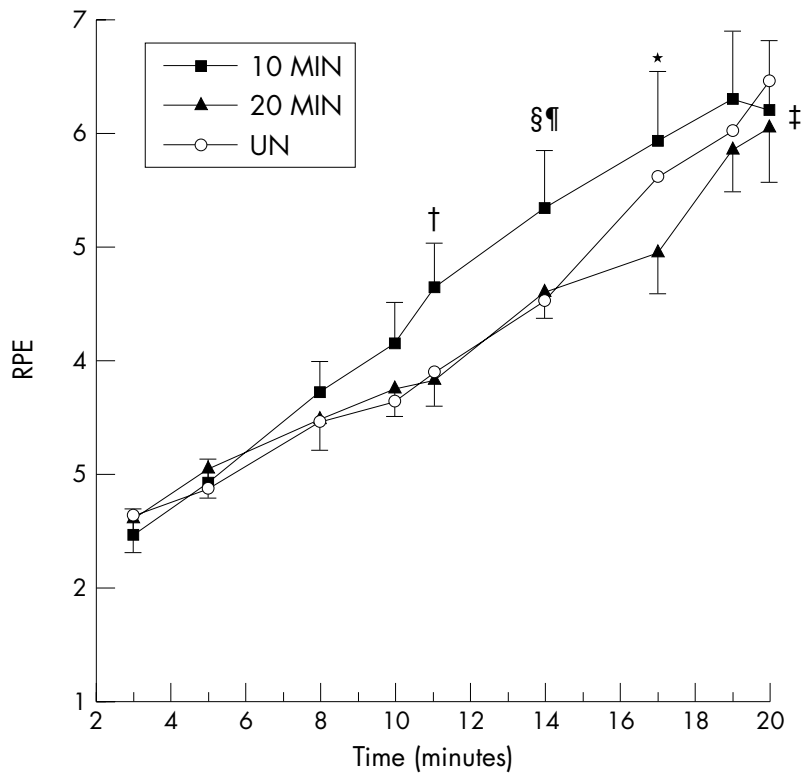

Figure 1 Rating of perceived exertion (RPE) during three trials: $20 \mathrm{MIN}$, subjects told to run for 20 minutes and stopped at 20 minutes; $10 \mathrm{MIN}$, subjects told to run for 10 minutes, but at 10 minutes told to run for a further 10 minutes; UN, subjects not told for how long they would be running but stopped after 20 minutes. Values are mean (SEM) ( $\mathrm{n}=$ 16). ¥Significant increase over time for all trials $(p<0.01)$. †Significant increase from 10 to 11 minutes in $10 \mathrm{MIN}$ trial $(p<0.05)$. *Significant difference between $10 \mathrm{MIN}$ and $20 \mathrm{MIN}$ trials $(\mathrm{p}<0.01)$. Significant difference between $10 \mathrm{MIN}$ and $20 \mathrm{MIN}$ trials $(p<0.05)$. §Significant difference between $10 \mathrm{MIN}$ and UN trials $(p<0.05)$.

\section{Experimental sessions}

Within one week of the familiarisation session and the PTRS test, subjects performed three trials in random order on a motorised treadmill (Quinton Instruments, Seattle, Washington, USA). In all three trials, the treadmill speed for each subject was set at $75 \%$ of their PTRS. In one trial, subjects were informed that they would run for 20 minutes and then completed a 20 minute run $(20 \mathrm{MIN})$. In the second trial, subjects were told that they would run for 10 minutes, but, at one minute from completion of the 10 minutes, were asked to run for another 10 minutes, so that the total exercise duration was 20 minutes (10 MIN). Exercise intensity was maintained at a constant level for the entire 20 minutes of the 10 MIN trial. In the third trial, subjects were not told for how long they would be running, but were stopped after 20 minutes had been completed (unknown trial, UN). Thus the total duration and intensity were the same for all three trials (20 minutes at $75 \%$ of PTRS), but subjects were informed differently about the expected exercise duration before the start of each trial. The same investigators were present during all trials, and the same level of encouragement was given to the subject in each of the three trials. Subjects performed the three experimental trials at the same time of day, so that effects of circadian rhythm on physiological and psychological function were negated, and at least a day of rest was allowed between each trial.

During each trial, RPE, affect, attentional focus (percentage associative thoughts), $\mathrm{VO}_{2}$, stride frequency, and heart rate were measured at $3,5,8,9,10,11,14,17,19$, and 20 minutes. The subjects were made aware of how much time had passed at each of these time points when these measurements were performed. The different length of time between each data point was deliberately chosen, as we expected changes to occur in the first minute after changes in

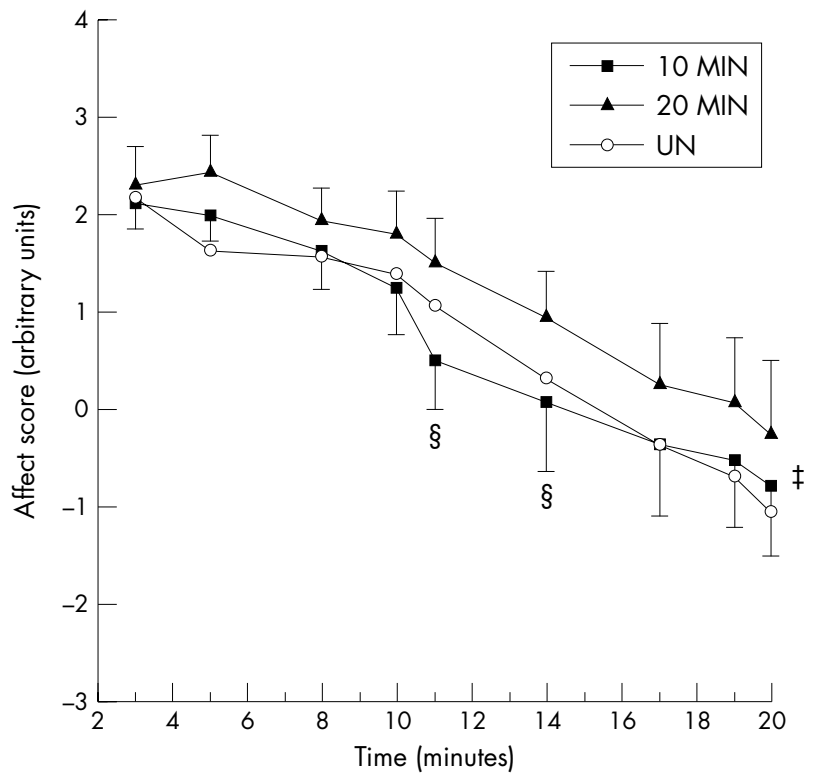

Figure 2 Affect scale scores during three trials: $20 \mathrm{MIN}$, subjects told to run for 20 minutes and stopped at 20 minutes; $10 \mathrm{MIN}$, subjects told to run for 10 minutes, but at 10 minutes told to run for a further

10 minutes; UN, subjects not told for how long they would be running but stopped after 20 minutes. Values are mean (SEM) $(n=16)$.

$\ddagger$ Significant decrease over time for all trials $(p<0.01)$. §Significant difference between $10 \mathrm{MIN}$ and $20 \mathrm{MIN}$ trials $(p<0.05)$.

the 10 MIN group at minute 10. Therefore, to assess these predicted changes, data were recorded at 9, 10, and 11 minutes into the trial. As we did not want to have different lengths of time only around the 10 minute time point, length of time between measurements was thus varied throughout the trial in an attempt to make the length of time between measurements appear random. However, the same time points were used for data measurement in all three trials.

\section{RPE}

RPE was measured during each trial at the specified times using the Borg category ratio scale. ${ }^{12}$ This scale measured the overall feelings of subjective sensation of effort accompanying exercise. The scale and what it measured was carefully explained to all subjects during the familiarisation session and before each experimental trial. It was emphasised that the scale measures physical strain or work, and instructions were given according to suggestions from Pandolf ${ }^{17}$ and Noble et al. ${ }^{2}$

\section{Affect scale}

Affect was measured using the affect (feeling) scale, developed and validated by Rejeski and Kenney. ${ }^{9}$ The affect scale is a bipolar scale which ranges from +5 to -5 with verbal descriptors: $+5=$ very good; $+3=$ good; $+1=$ slightly good; $0=$ neutral; $-1=$ slightly bad; $-3=$ bad; $-5=$ very bad. ${ }^{9}$

Subjects were informed and subsequently reminded that the scale measured the affective or emotional component of exercise, such as whether the sensation of effort during the treadmill running bout felt pleasant or unpleasant, and not the actual level of physical effort or strain.

\section{Associative thought scale}

During each trial, subjects were asked to report what percentage of their thoughts were associative thoughts. 


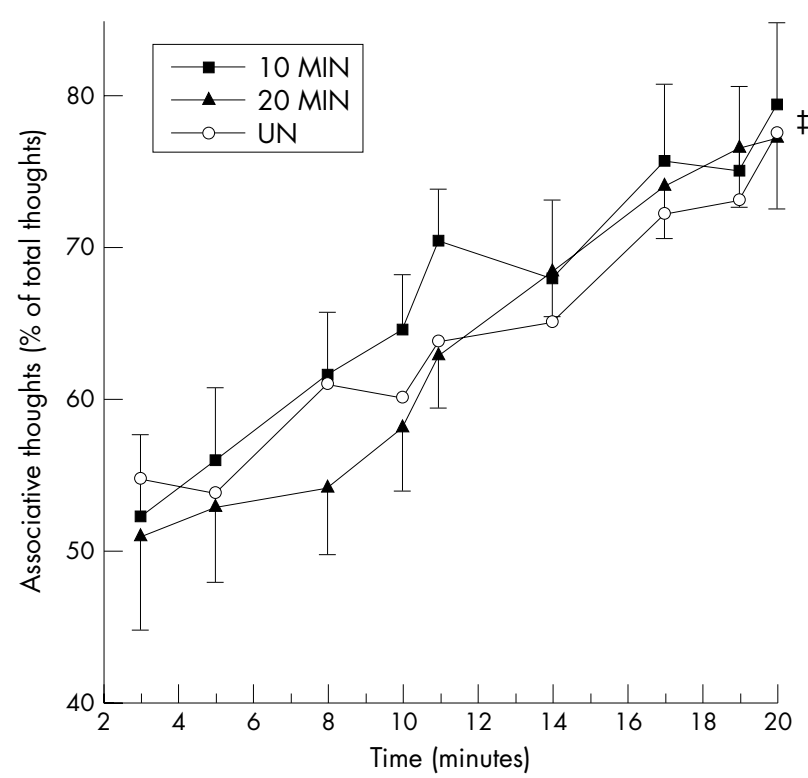

Figure 3 Associative thoughts percentage during three trials: $20 \mathrm{MIN}$, subjects told to run for 20 minutes and stopped at 20 minutes; $10 \mathrm{MIN}$, subjects told to run for 10 minutes, but at 10 minutes told to run for a further 10 minutes; UN, subjects not told for how long they would be running but stopped after 20 minutes. Values are mean (SEM) $(n=16)$. $\ddagger$ Significant increase over time for all trials $(p<0.01)$.

Associative thoughts were defined as those directed towards bodily symptoms, and measured as a score between 0 and 100, with 100 representing only associative thoughts. Dissociative thoughts were defined as thoughts that distract the subjects from the exercise being performed and directed towards external factors, ${ }^{18}$ and were measured as the remainder of the thought score not described as associative thoughts.

\section{Oxygen consumption}

$\mathrm{VO}_{2}$ was measured continuously during all trials using an automated gas analysis system (Oxycon Alpha; Enrich Jaeger, Wuerzburg, Germany), as described previously. ${ }^{10}$

\section{Heart rate}

Heart rate was measured during all trials using a Polar S410 heart rate monitor (Polar Electro Oy, Kempele, Finland), as described previously. ${ }^{10}$

\section{Stride frequency}

The number of times the right foot of the subject landed on the treadmill was counted during the 30 seconds leading up to the specified time points, and this value was doubled to give a measurement of stride frequency $\left(\min ^{-1}\right)$.

\section{Statistical analysis}

All data were analysed using a Statistica (StatSoft Inc, Tulsa, Oklahoma, USA). A repeated measures analysis of variance was conducted to compare data over all three trials ( 10 MIN, $20 \mathrm{MIN}$, and UN). Where a significant interaction effect was found, post hoc analyses were conducted using a Tukey HSD post hoc test. Data are presented as mean (SEM). An $\alpha$ level of $\mathrm{p}<0.05$ was taken as significant.

\section{RESULTS}

\section{Subject characteristics}

The mean (SEM) age of the subjects was 30.4 (4.1) years, mass $68.0(12.2) \mathrm{kg}$, height $173(7) \mathrm{cm}$, and percentage body fat $20.8(4.4) \%$. The $\mathrm{VO}_{2}$ peak was $56.4(2.9) \mathrm{ml} / \mathrm{kg} / \mathrm{min}$, PTRS
18.1 (1.5) $\mathrm{km} / \mathrm{h}$, and mean distance run each week 31.9 (13.3) $\mathrm{km}$.

\section{RPE}

RPE increased essentially linearly with increasing exercise duration in all three trials (fig 1). However, RPE increased significantly more between 10 and 11 minutes in the 10 MIN trial compared with the 20 MIN trial $(p<0.05)$, so that RPE at 11 minutes was significantly higher in the 10 MIN trial than in the 20 MIN trial $(p<0.05)$. RPE remained significantly higher in the 10 MIN trial than in the 20 MIN trial until 17 minutes $(\mathrm{p}<0.05)$. RPE was also higher in the 10 MIN trial than in the UN trial from 11 minutes to 14 minutes (fig 1).

\section{Affect scale scores}

Affect scale scores decreased significantly (affect became more negative) over the course of the trial in all three conditions $(\mathrm{p}<0.01$, fig 2 ). However, the affect score fell more steeply between 10 and 11 minutes during the 10 MIN trial than during the $20 \mathrm{MIN}$ and UN trials. As a result, the affect score during the 10 MIN trial was significantly lower than during the $20 \mathrm{MIN}$ and UN trials at 11 minutes and 14 minutes (fig 2). Affect score was higher throughout the 20 MIN trial than during both the 10 MIN and UN trials.

\section{Associative thoughts}

The percentage of associative thoughts increased significantly during all three trials $(\mathrm{p}<0.01$, fig 3$)$. No significant differences were found between trials, although the percentage change tended to be higher in the 10 MIN trial between 10 and 11 minutes compared with the 20 MIN and UN trials.

\section{Oxygen consumption}

$\mathrm{VO}_{2}$ was significantly lower during the UN trial than during the 20 MIN trial from 10 minutes until 19 minutes $(\mathrm{p}<0.05$,

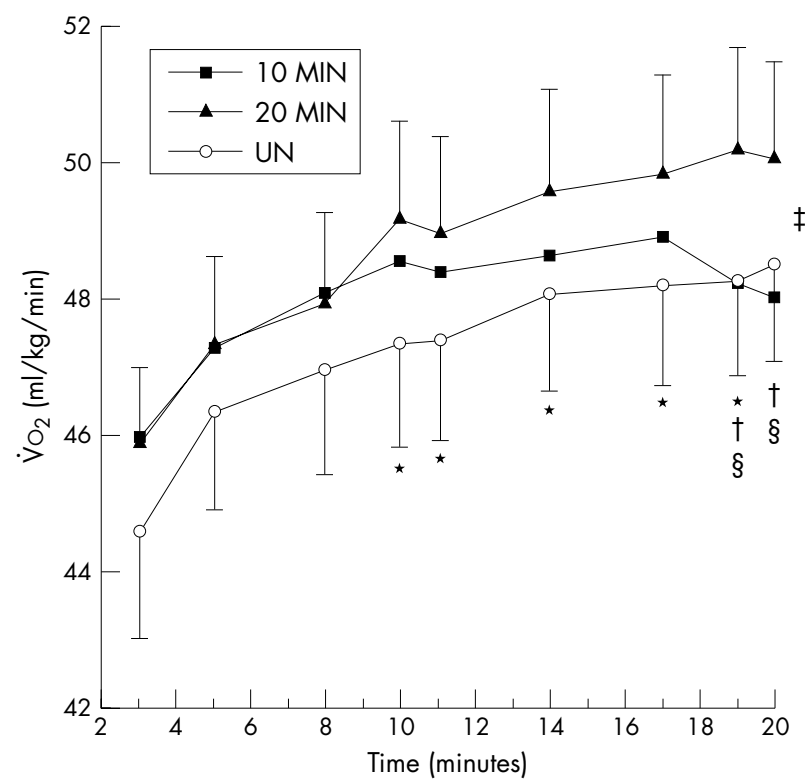

Figure 4 Oxygen consumption $\left(\mathrm{VO}_{2}\right)$ during three trials: $20 \mathrm{MIN}$, subjects told to run for 20 minutes and stopped at 20 minutes; $10 \mathrm{MIN}$, subjects told to run for 10 minutes, but at 10 minutes told to run for a further 10 minutes; UN, subjects not told for how long they would be running but stopped after 20 minutes. Values are mean (SEM) $(n=16)$. $\ddagger$ Significant increase over time for all trials $(p<0.01)$. *Significant difference between UN and 20 MIN trials $(p<0.05)$. †Significant difference between $U N$ and $10 \mathrm{MIN}$ trials $(p<0.05)$. §Significant difference between $10 \mathrm{MIN}$ and $20 \mathrm{MIN}(p<0.05)$. 
fig 4). $\mathrm{VO}_{2}$ was lower during the UN trial than the 10 MIN trial at minute 3, and remained significantly lower from minute 9 until 17 minutes. $\mathrm{VO}_{2}$ during the $10 \mathrm{MIN}$ trial decreased from 17 minutes, and was significantly lower than the 20 MIN trial at 19 and 20 minutes ( $<<0.05$, fig 4 ).

\section{Heart rate}

Heart rates increased significantly over time in all three trials $(\mathrm{p}<0.01)$, but were not different between the trials.

\section{Stride frequency}

Stride frequency was not different between trials at any time point, and did not change significantly during any of the trials.

\section{DISCUSSION}

The first important finding of this study was that when subjects were deceived during the $10 \mathrm{MIN}$ trial and were told near the end of the expected trial duration to run for 10 minutes longer than anticipated, RPE increased significantly at 11 minutes, compared with the 20 MIN trial, before which subjects had been honestly informed of the correct duration of the run (fig 1). Exercise intensity (75\% of PTRS for all trials), $\mathrm{VO}_{2}$ (fig 4), heart rate, and stride frequency were not significantly different from 10 to 11 minutes in any of the trials. The physiological demands of the running task did not therefore change at the same stage as changes in RPE were observed. The increase in RPE consequent to misinformation about the expected exercise duration was therefore not caused by changes in physiological factors. Therefore we suggest that the change in RPE at 11 minutes in the 10 MIN trial, which was significantly higher than in the other trials until 14 minutes, was the result of affective processes, responding to the unexpected sudden increase in exercise duration or the sudden imposition of a mismatch between the previously anticipated and the new required exercise duration.

Indeed, affect scale scores, which are a measure of the emotional perception of the cognitive sensations induced by the exercise bout, decreased significantly at 11 minutes in the 10 MIN trial, but not in the 20 MIN or UN trial (fig 2). The increased and significantly higher RPE in the 10 MIN trial may therefore be attributed to a decrease in positive affect when anticipations of the exact exercise duration were not met. This indicates that the perception of exertion is closely associated with affect. Indeed, Pennebaker ${ }^{19}$ showed that the perception of bodily symptoms is influenced not only by physiological but also by psychological processes. Williamson et $a l^{20}$ showed that hypnotic manipulation affected effort sense and its relation to heart rate and blood pressure responses. Interestingly, in their study, hypnotic induced increases in the sense of effort during constant load exercise were associated with increase in heart rate and blood pressure, whereas hypnotic induced decreases in the sense of effort were not associated with changes in either heart rate or blood pressure. The effect of hypnotic manipulation and alteration of end point manipulation therefore produced different results, as increased RPE and affect in our trial were not associated with increases in heart rate. The reasons for these differences are not immediately clear, but it would be

\section{What is already known on this topic}

Previous work has suggested that perceived exertion during exercise is causally linked to changes in running speed and physiological variables such as heart rate, ventilation rate, and blood lactate concentrations. interesting to examine the changes in affect associated with hypnotic manipulation, as these were not measured during the trial of Williamson et $a^{20}$ and may be a cause of the different findings in the two studies.

Pohl et $a^{21}$ have suggested that subjects will display symptoms to match their expectations in a process termed symptom belief. That is, expectations have a large influence on perceived symptoms, which in the present study would include the RPE during the running task. When expectations of a task are not met, as occurs in the $10 \mathrm{MIN}$ trial, subjects may consciously or unconsciously experience negative affect, including emotions such as anger, frustration, distrust, and doubt, responses that are reflected in the affect scale scores in this study (fig 2). St Clair Gibson et al ${ }^{22}$ have proposed that the knowledge of the physiological processes associated with the fatigue process initially occurs at the subconscious, cognitive level and that feelings of affective responses are the conscious representations of these subconscious changes. The link between the increase in RPE and the decrease in affect score in the absence of changes in physiological variables or exercise intensity supports the hypothesis that fatigue, commonly thought to be a physical process, may rather be an emotional construct.

A further finding was that associative thoughts percentage shows a significant increase over time for all trials (fig 3). Progressive increases in the percentage of associative thoughts suggest that the subjects have a narrower attention focus. That is, as the trial progresses, subjects engage in thoughts more related to the task of running. Although no significant differences in associative thoughts were found between trials, there was a tendency for the percentage associative thoughts to decrease from 11 to 14 minutes in the 10 MIN trial, immediately after a sharp increase from 10 to 11 minutes. This pattern of change may indicate that subjects attempted to reset their focus in order to complete the trial, or as an attempt to improve the reduced affect caused by the instruction to continue for a further 10 minutes (fig 2). These changes in associative thoughts occurred at the same time as an increase in RPE (fig 1) and a decrease in feeling score (fig 2), as discussed previously.

The next important finding was that $\mathrm{VO}_{2}$ during the UN trial was lower than in the 20 MIN trial throughout, with significant differences from 10 minutes until 19 minutes (fig 4). At the same exercise intensity, a lower $\mathrm{VO}_{2}$ suggests improved running economy, ${ }^{23}$ and the lower $\mathrm{VO}_{2}$ in the UN trial supports the hypothesis that when a task of unknown duration is performed, subjects will be more economical in their use of physiological resources, in order to maintain a reserve in anticipation of a longer exercise bout and greater physiological demand. The mechanism for this improvement is not clear, and the data do not allow us to conclude which factors may be responsible for the measured improvement in running economy. Running economy has previously been related to heart rate and biomechanical variables. ${ }^{24-26}$ Neither heart rate nor stride frequency showed any significant differences between the three trials, suggesting that more subtle changes, such as changes in muscle recruitment ${ }^{14}$ which were not measured in the present study, may underlie this finding.

\section{What this study adds}

This study shows that perceived exertion during exercise may rather be a psychological construct, which is scaled using knowledge of the exercise duration, and altered by changes in affect and cognitive focus of the athlete. 
In a study to measure the effect of psychological state on running economy, Williams et $a^{27}$ found that less negative affect was associated with lower $\mathrm{VO}_{2}$ for a given workload. We did not observe such an association, as affect was least affected in the 20 MIN trial (fig 2) but this trial did not produce the lowest $\mathrm{VO}_{2}$ (fig 4). However, that study used the profile of mood states scale to determine mood state of the subjects during the week before a run, whereas we used the feeling scale. Mood has been defined as a more stable measure of emotion, whereas affect is a more immediate change in emotion, ${ }^{7}$ and the use of two different scales to assess two different constructs may account for this finding.

An interesting observation was that the $\mathrm{VO}_{2}$ during the 10 MIN trial decreased after 17 minutes, and was significantly lower in the 10 MIN trial than in the 20 MIN trial at 19 and 20 minutes (fig 4). This may indicate that subjects become more economical as the end of the second 10 minute period of the 10 MIN trial approached, because of their experience a few minutes earlier of having been deceived. If they anticipated a second deception and the possibility that they would be told to continue exercise for another 10 minutes, they might have chosen to become more economical. It is noteworthy that the 10 MIN trial essentially becomes a trial of uncertain duration after the first 10 minutes, as subjects will no longer trust the experimenter. Thus the decrease in $\mathrm{VO}_{2}$ at the end of the 10 MIN trial agrees with the suggestion that subjects adopt a more economical running strategy when the duration of exercise is unknown.

In conclusion, in this study we found that unknown exercise duration and an unexpected increase in exercise duration influenced RPE, affect, and $\mathrm{VO}_{2}$. RPE appeared to be influenced by affect, and was not merely the result of a direct interpretation of the physiological changes occurring in different metabolic systems, as RPE increased significantly after an unexpected increase in running duration in the absence of changes in exercise intensity, $\mathrm{VO}_{2}$, or heart rate in this trial. Further research is required to determine what specific emotions correlate with perception of effort and running economy during exercise.

\section{ACKNOWLEDGEMENTS}

Funding for this experiment was provided by the Medical Research Council of South Africa, the University of Cape Town Harry Crossley and Nellie Atkinson Staff Research Funds, Discovery Health and the National Research Foundation of South Africa through the THRIP initiative.

\author{
Authors' affiliations \\ D A Baden, University of Southampton, Southampton, Hampshire, UK \\ T L McLean, R Tucker, T D Noakes, A St Clair Gibson, University of Cape \\ Town, Research Unit for Exercise Science and Sports Medicine, \\ Newlands, South Africa \\ Competing interests: none declared
}

\section{REFERENCES}

1 Borg GA. Psychophysical bases of perceived exertion. Med Sci Sports Exerc 1982;14:377-81.
2 Noble BJ, Borg GA, Jacobs I, et al. A category-ratio perceived exertion scale: relationship to blood and muscle lactates and heart rate. Med Sci Sports Exerc 1983;15:523-8.

3 Cafarelli E. Peripheral contributions to the perception of effort. Med Sci Sports Exerc 1982; 14:382-9.

4 Robertson RJ. Central signals of perceived exertion during dynamic exercise. Med Sci Sports Exerc 1982;14:390-6.

5 Rejeski WJ, Ribisl PM. Expected task duration and perceived effort: an attributional analysis. J Sport Psychol 1980;39:249-54.

6 Walster B, Aronson E. Effect of expectancy of task duration on the experience of fatigue. J Exp Soc Psychol 1967;3:41-6.

7 Crews DJ. Psychological state and running economy. Med Sci Sports Exerc 1992;24:475-82.

8 Hardy CJ, Rejeski WJ. Not what, but how one feels: the measurement of affect during exercise. Journal of Sports and Exercise Psychology 1989;1 1:304-17.

9 Rejeski WJ, Kenney E. Distracting attentional focus from fatigue: does task complexity make a difference? J Sport Psychol 1987;9:66-73.

10 Ansley L, Robson PJ, St Clair Gibson A, et al. Evidence for anticipatory strategies during supra-maximal exercise lasting longer than 30s. Med Sci Sports Exerc 2004;36:309-14.

11 Baden D, Warwick-Evans LA, Lakomy J. Am I nearly there? The effect of anticipated running distance on perceived exertion and attentional focus. Journal of Sports and Exercise Psychology 2004;27:215-31.

12 St Clair Gibson A, Noakes TD. Evidence for complex system integration and dynamic neural regulation of skeletal muscle recruitment during exercise in humans. Br J Sports Med 2004;38:797-806.

13 Ulmer H-V. Concept of an extracellular regulation of muscular metabolic rate during heavy exercise in humans by psychophysiological feedback. Experientia 1996;52:416-20.

14 Vidacek S, Wishner J. Influence of expectation of task duration on efficiency of muscular activity. J Appl Psychol 1971;55:564-9.

15 Durnin JV, Womersley J. Body fat assessed from total body density and its estimation from skinfold thickness: measurements on 481 men and women aged from 16 to 72 years. Br J Nutr 1974;32:77-97.

16 Sharwood KA, Lambert Ml, St Clair Gibson A, et al. Changes in oxygen consumption during and after a downhill run in masters long-distance runners. Clin J Sports Med 2002;12:308-12.

17 Pandolf KB. Differentiated ratings of perceived exertion during physical exercise. Med Sci Sports Exerc 1982;14:397-405.

18 Morgan WP, Pollock ML. Psychological characterization of the elite distance runner. Ann NY Acad Sci 1997;301:382-403.

19 Pennebaker JW. The psychology of physical symptoms. New York: Springer Verlag, 1982.

20 Williamson JW, McColl R, Mathews D, et al. Hypnotic manipulation of effort sense during dynamic exercise: cardiovascular responses and brain activation. J Appl Physiol 2001;90:1392-9.

21 Pohl J, Frohnau G, Kerner W, et al. Symptom awareness is affected by the subjects' expectations during insulin-induced hypoglycemia. Diabetes Care 1997;20:796-802.

22 St Clair Gibson A, Baden DA, Lambert MI, et al. The conscious perception of the sensation of fatigue. Sports Med 2003;33:167-76.

23 Morgan DW. Factors affecting running economy. Sports Med 1989;7:310-30.

24 Conley D, Krahenbuhl G. Running economy and distance running performance of highly trained athletes. Med Sci Sports Exerc 1980;12:357-60.

25 Cavanagh $\mathbf{P}$, Williams $\mathrm{K}$. The effect of stride length variation on oxygen uptake during distance running. Med Sci Sports Exerc 1982;14:30-5.

26 Williams K, Cavanagh P. Relationship between distance running mechanics running economy, and performance. J Appl Physiol 1987;63:1236-45.

27 Williams TJ, Krahenbuhl G, Morgan DW. Mood state and running economy in moderately trained male runners. Med Sci Sports Exerc 1991;23:727-31.

\section{COMMENTARY}

This paper has come up with some fundamental questions that exercise scientists have ignored for far too long. The implications of these data will be far reaching in pushing the scientific boundaries further.

A M Hunter

Department of Sports Studies, University of Stirling, Stirling, UK; a.m.hunter1@stir.ac.uk 